\title{
Giant cholesterol granuloma of the petrous apex: endoscopic management
}

\begin{abstract}
Cholesterol granulomas are rare and benign lesions that can be found anywhere in the body. These lesions can be seen indifferent locations in the temporal bone such as in the petrous apex, middle ear and mastoid. We present a case report of a petrous apex cholesterol granuloma in a 69years old male with an atypical peripheral facial palsy, sensorineural hearing loss and tongue paresthesias. CT and MRI studies are essential for the differential diagnosis and the selection of the ideal surgical technique to be performed. Due to its safety and little morbidity, a trans-sphenoidal endoscopic approach was made.
\end{abstract}

Keywords: cholesterol, granuloma, petrous, apex, fees, endoscopic, sinus, surgery
Volume 10 Issue I - 2018

\author{
Fernandez Manzano C,' Pinilla Urraca M,' \\ Rijo-Cedeño J,' Arellano B,' Brea B, ${ }^{2}$ Bellas \\ Menendez $\mathrm{C}^{3}{ }^{3}$ López-Cortijo $\mathrm{C}^{\prime}$ \\ 'Department of Otorhinolaryngology, Puerta de \\ HierroMajadahonda, University Hospital, Universidad Autonoma \\ Madrid, Spain \\ 2Department of Neuroradiology, Puerta de HierroMajadahonda, \\ University Hospital, Universidad Autonoma Madrid, Spain \\ ${ }^{3}$ Department of Anatomopathology, Puerta de \\ HierroMajadahonda, University Hospital, Universidad Autonoma \\ Madrid, Spain
}

\author{
Correspondence: Fernandez Manzano Cristobal Hospital \\ Universitario Puerta de Hierro C Manuel de Falla I 28222 \\ Majadahonda Madrid, Spain, Tel +34 66161 I044, \\ Email criferman@gmail.com
}

Received: November 21, 2017 | Published: February 02, 2018
Abbreviations: CT, computed tomography; MRI, magnetic resonance imaging; COPD, chronic obstructive pulmonary disease; PA, petrous apex; FESS, functional endoscopic sinus surgery ; FEESST, flexible endoscopic evaluation of swallowing with sensory testing

\section{Introduction}

Cholesterol granulomas are rare and benign lesions characterized for having a cystic pattern containing cholesterol crystals that are usually surrounded by foreign body giant cells. Their growing pattern is very variable and it is related to the frequency and severity of the micro hemorrhages happening within. It can be found in different locations in the temporal bone such as in the petrous apex (PA), middle ear and mastoid.

Most of these temporal bone lesions take decades to grow and symptoms show up years after an initial history of chronic otitis media.

It normally appears as an encapsulated cyst containing a brown sand-like fluid formed by cholesterol crystals and other elements from the blood degradation. It is more frequent in young to middleage adults.

The clinical presentation may vary depending on the extension and location of the mass. In order of frequency, the most common signs and symptoms of a petrous apex cholesterol granuloma ${ }^{1}$ are sensorineural hearing loss, vestibular symptoms, tinnitus, facial twitching, facial paresthesia, diplopia and facial weakness. Otoscopy is usually normal, but a blue retrothympanic occupation may be seen if the lesion has gotten into the middle ear.

In a computed tomography scan it can be seen as an avascular image, isodense to the cerebral parenchyma, with mastoid erosion. This allows to discard most of the expansive lesions of the petrous apex with the exception of mucoceles and cholesteatomas. In order to differentiate between cholesterol granuloma and cholesteatoma it is necessary to perform a magnetic resonance (MR), where cholesterol granuloma is seen as a smooth, sharply marginated and lobulated mass, hyper intense in bothT1, T2 and FLAIR signal. Cholesteatomas appear as a hypointense mass in T1 signal with reinforcement in T2.

Asymptomatic patients can be safely followed up with image testing. The traditional treatment in the symptomatic ones is the drainage and stent placement via a translabyrinthine, infralabyrinthine, middle fosa, trans-sphenoidal or infracochlear approach to re-establish PA aeration.

The reported recurrence rate is a $14-16 \%$. The extended middle cranial fossa approach with extradural removal and obliteration of the cavity gives a significant decrease in recurrence rate. However, in the cases where It's possible, it should be performed a trans-sphenoidal endoscopic surgery due to its lower morbidity.

Long-term success of the surgical management is related to the maintenance of PA drainage, causing a consequently gradual reduction of the cavity size.

\section{Case presentation}

A 69year old male came to the emergency room referring left facial palsy with ipsilateral sensitive tongue disturbances associated. He also referred left ear hearing loss since a few years beforewhich had increased in the last two months. Personal background: active smoker and alcohol drinker, arterial hypertension, psoriasis, COPD.

The patient was treated with oral corticosteroids (Prednisone $1 \mathrm{mg}$ per kilogram and day in descending the dose gradually) for two weeks with resolution of the facial palsy but persistence of the other symptoms.

Physical examination showed a normal otoscopy, tongue and facial motility, preserved nausea reflex (examined with FEESST), hyposensitivity in left hemi tongue and normal otoneurologic examination. 


\section{Complementary tests}

Audiometry: Moderate-Severe left sensorineural hearing loss

Brain MR and CT (Figure 1): Showed an expansive lesion in the left petrous apex, with well-defined contours and hyperintense signal in T1 and hypointense areas in T2, eroding the facial nerve canal, inner acoustic canal and part of the cochlea. The lower part the lesion was eroding the medial wall of the carotid canal. Additionally, it was spreading to the skull base expanding the body of sphenoid bone, sphenoidal sinus and ethmoidal cells and displacing the pituitary gland upwards.
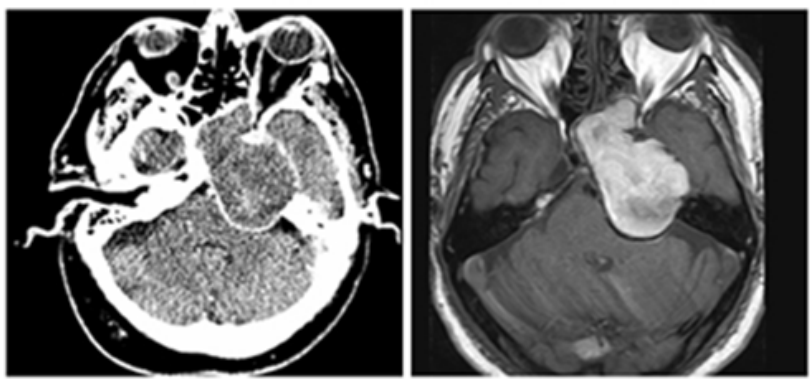

Figure I Axial CT and TI-weighted MR image showing an expansive process in the left petrous apex.

It also invaded the pontocerebellar area and conditioned a mass effect over the pons of the brainstem. Because of all these signal properties as well as it location the lesion was suggestive of cholesterol granuloma as the first diagnostic probability.

Treatment: An endoscopic approach with sphenoidotomy was performed, showing a pulsatile encapsulated mass (Figure 2). After the aperture of the capsule, samples of the lesion were taken and a big part of the mass was aspirated. During drainage of the mass content the patient suffered fluctuations in his heart rhythm, thus a complete emptying of the mass was not done in order to avoid decompression of the brainstem and the possible risk of cerebral hernia.

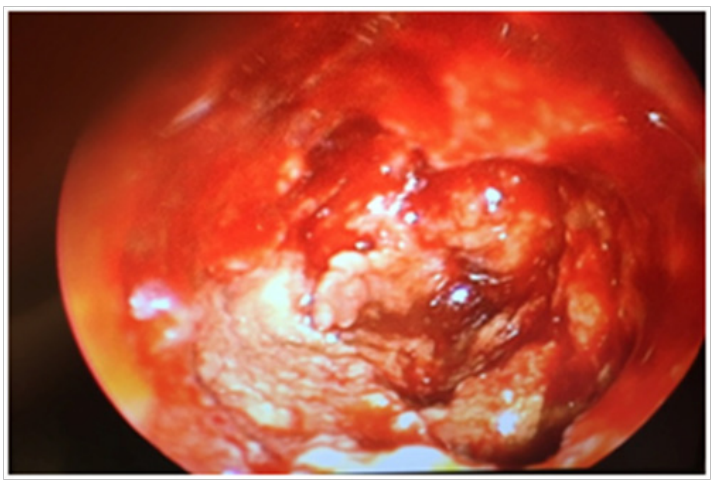

Figure 2 Endoscopic sphenoidotomy showing the pulsatile encapsulated mass.

Final diagnosis: The histopathological analysis confirmed the suspicion of cholesterol granuloma and demonstrated abundant cholesterol clefts with giant cells around them and inflammatory cells (Figure 3).

Evolution:There were no postoperative incidences. MR from 7 days after surgery (Figure 4) showed a decrease of the lesion size compared with the preoperative image observing a more heterogeneous content in $\mathrm{T} 1$ signal and air bubbles in relation to the recent surgery. During the 2-year follow-up the patient was asymptomatic and stable, requiring no additional endoscopic neurosurgical treatment.

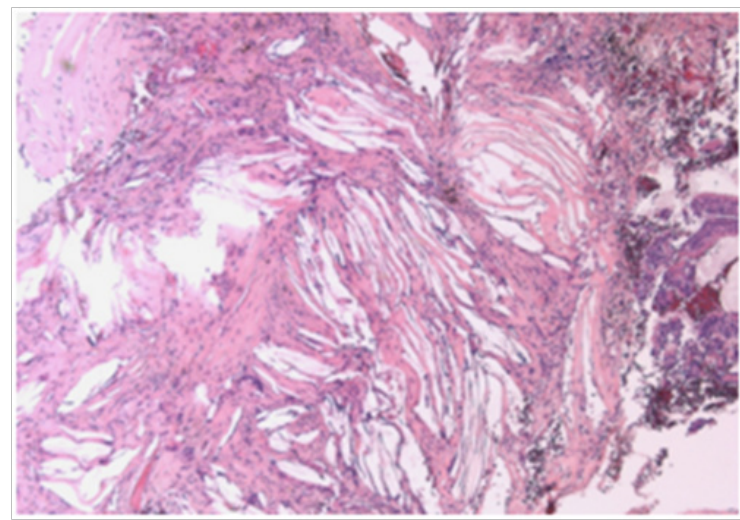

Figure 3 Cholesterol clefts with giant cells around them and inflammatory cells.

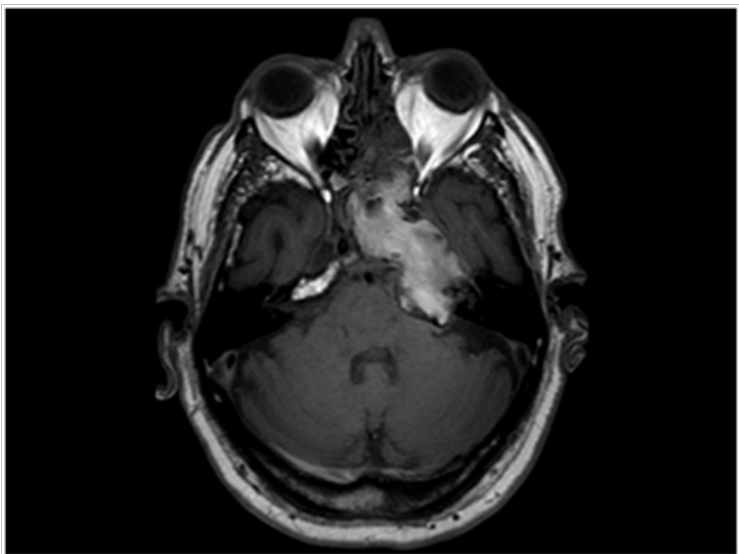

Figure 4 Postoperative axialT2-weighted MR image showing a decrease of the lesion size compared with the preoperative image observing a more heterogeneous content in TI signal and air bubbles in relation with the recent surgery.

\section{Discussion}

A cholesterol granuloma is a benign lesion caused by a giant cell inflammatory reaction to blood degradation products that act as a foreign body. Although benign, it can behave aggressively depending on the anatomical location and the relation with neurovascular structures. This pathology can arise in different regions of the temporal bone such as in the petrous apex, middle ear or mastoid.

There are two main theories about its origin. The first one ${ }^{2}$ suggests the mucosa covering the petrous apex air cells occludes the cavity and leads to a negative pressure and posterior hemorrhage. Due to the degradation of the blood elements, cholesterol crystals precipitate and stimulate an inflammatory granulomatous reaction. With time an expansive progression is produced and induces an erosion of the bone.

The second theory, developed by Jackler \& Cho, ${ }^{3}$ hypothesize that a highly pneumatized temporal bone exposes marrow-filled spaces of the petrous apex and coats the mucosa producing a subacute hemorrhage.

A peripheral facial palsy requires a complete anamnesis and physical exploration, as well as complementary image tests in cases of atypical Bell's palsy in order to discard expansive tumors of the cerebello pontine angle. 
Radiological differential diagnosis ${ }^{4}$ of the cholesterol granuloma of the petrous apex includes congenital pathologies such as congenital cholesteatoma, inflammatory diseases(apical petrositis, mucocele), benign tumors (meningioma, shwannoma, paraganglioma), malign tumors (lymphoma, metastatic disease, myeloma) metabolic and dysplastic diseases such as Paget disease and also Histiocytosis $\mathrm{X}$ (Table 1).

The most typical radiological presentation in the MRI is an expansive lesion in the petrous apex, with well-defined contours and
T1 hyperintense signal with $\mathrm{T} 2$ hypointense areas, as it was seen in our patient.

Regarding the treatment, there are two different approaches according to whether the patient is symptomatic or not ${ }^{5}$. In asymptomatic patients a follow up (or wait and see) strategy is recommended with periodic radiological evaluations (using CT or MR). In the symptomatic patients the surgical treatment is the best option.

Table I Radiological differential diagnosis features

\begin{tabular}{llll}
\hline & CT & MR & \\
\hline & & TI & T2 \\
\hline Cholesterol Granuloma & Isodense & Hyperintense & Hyperintense \\
\hline Epidermoid Cyst & Hypodense or Isodense & Hypointense & Hyperintense \\
\hline Arachnoid Cyst & Hypodense & Hypointense & Hyperintense \\
\hline Apical Petrositis & Hypodense & Hypointense & Hyperintense \\
\hline Congenital cholesteatoma & Isodense & Hypointense & Hyperintense \\
\hline
\end{tabular}

The best surgical approach should be chosen considering the location of the lesion, its extension, relationship with neurovascular structures, anatomical variations and previous hearing status.

A translabyrinthine, infralabyrinthine, middle fossa, trahpssphenoidal or infracochlear approach can be chosen depending on the characteristics of the patient.

The most important aspect for the long-term success of the surgical management is the maintenance of a drainage pathway (in some cases with stent placement) to re-establish PA aeration to allow a gradual reduction of the cavity size.

Some authors ${ }^{6}$ defend that the extended middle cranial fossa approach with extradural removal and obliteration of its cavity make a significant decrease in recurrence rates. The recurrence rate reported in the literature ${ }^{7}$ is up to $14 \%-16 \%$ and the failure of the procedure can be attributed to the early closure of the drainage pathway.

Transsphenoidal approach was first described in 1977 by Montgomery $^{8}$ as an external procedure through an incision near the medial canthus. Endoscopic approach was not described until 1994, when Fucci ${ }^{9}$ adapted the use of the nasal endoscope as an approach to this lesion.

Endonasal endoscopic removal of cholesterol granuloma is less invasive than the previous middle and lateral fossa approach procedures and has several advantages; no risk of lesion of facial nerve or vestibule-cochlear function and a reduced operative time and hospitalization. It also can be easily followed up with office fiber-optic examination. Because of all these reasons we decided to perform this approach. ${ }^{10-12}$

\section{Conclusion}

Although benign, petrous apex cholesterol granulomas can behave aggressively due to its location. It can cause a great deal of different symptoms because of its proximity to encephalic structures. A thorough clinical examination should be performed and image testing, specially MRI, are essential not just for the diagnosis but also for a correct surgical planning.

\section{Acknowledgements}

None.

\section{Conflicts of interest}

The authors declare no conflict of interest or external financing.

\section{Funding}

None.

\section{References}

1. Hoa M, House JW, Linthicum FH, et al. Petrous apex cholesterol granuloma: pictorial review of radiological considerations in diagnosis and surgical histopathology. J Laryngol. 2013;127(4):339-348.

2. Graham M, Kemnik J, Latack J, et al. The Giant cholesterol cystof the petrous apex. Laryngoscope. 1985;95(11):1401-140.

3. Jackler R, Cho M. A New Theory to Explain the Genesis of Petrous Apex Cholesterol Granuloma. Otology \& Neurotology. 2003; 24(1):96-106.

4. RicHarnsberger H, Christine MG, Michelle AM, et al. Diagnostic Imaging: Head and Neck. $1^{\circ}$ Ed. Salt Lake City: Amirsys, USA. 2004.

5. Sanna M, Dispenza F, Mathur N, et al. Otoneurological management of petrous apex cholesterol granuloma. Am $J$ Otolaryngol. 2009;30(6):407-414.

6. Cristante L, Puchner M. A keyhole middle fossa approach to large cholesterol granulomas of the petrous apex. Surg Neurol. 2000;53(1):64-71

7. Brackmann D, Toh E. Surgical Management of Petrous Apex Cholesterol Granulomas. Otology \&Neurotology. 2002;23(4):529-533.

8. Montgomery W. Cystic Lesions of the Petrous Apex: Transsphenoid Approach. Annals of Otology, Rhinology \& Laryngology. 1977;86(4):429-435.

9. Fucci M, Alford E, Lowry L, et al. Endoscopic Management of a Giant Cholesterol Cyst of the Petrous Apex. Skull Base Surg. 1994;4(1):52-58.

10. Jaberoo MC, Hassan A, Pulido MA, et al. Endoscopic Endonasal Approaches to Management of Cholesterol Granuloma of the Petrous Apex. Skull Base. 2010;20(5):375-379.

11. Georgalas C, Kania R, Guichard J, et al. Endoscopic transsphenoidal surgery for cholesterol granulomas involving the petrous apex. Clinical Otolaryngology. 2008;33(1):38-42.

12. Jaime Bermeo S, Víctor Mercado M, Fernando Ferrer R, et al Cholesterol granuloma. Present diagnostic and therapeutic approach. Rev otorrinolaringol cir cab-cuello . 2001;61:55-56. 